\title{
Prognostic significance of vascular and valvular calcifications in low- and high-gradient aortic stenosis
}

\author{
Brahim Harbaoui ${ }^{1,2 * \dagger}$, Nina Ghigo ${ }^{1 \dagger}$, Loic Boussel ${ }^{1,3 \dagger}$, Hervé Liebgott ${ }^{1 \dagger}$, \\ Géraud Souteyrand $^{4 \dagger}$, Eric Durand ${ }^{5 \dagger}$, Hélène Eltchaninoff ${ }^{5 \dagger}$, Thierry Lefevre ${ }^{6 \dagger}$, \\ Pierre-Yves Courand ${ }^{1,2 \dagger}$, and Pierre Lantelme $\mathbb{1}^{1,2 \dagger}$
}

\begin{abstract}
${ }^{1}$ University of Lyon, CREATIS UMR5220, INSERM U1044, INSA-15, 7 avenue Jean Capelle 69621 Villeurbanne Cedex, Lyon, France; ${ }^{2}$ Cardiology Department, Hôpital CroixRousse and Hôpital Lyon Sud, Hospices Civils de Lyon, Lyon, France; ${ }^{3}$ Radiology Department, Hôpital Croix-Rousse, Hospices Civils de Lyon, Lyon, France; ${ }^{4}$ Department of Cardiology, Gabriel Montpied University Hospital Center, Image Science for Interventional Techniques, Cardiovascular Interventional Therapy and Imaging, National Scientific Research Center UMR 6284, University of Auvergne, Clermont-Ferrand, France; ${ }^{5}$ Cardiology Service, Rouen-Charles-Nicolle University Hospital Center, National Institute of Health and Medical Research U644, Rouen, France; and 'Institut Cardiovasculaire Paris Sud, Ramsay-Générale de Santé, 6 avenue du Noyer Lambert, 91300 Massy, France
\end{abstract}

Received 24 November 2020; editorial decision 16 February 2021; accepted 17 February 2021

Aims

In low-gradient aortic stenosis (LGAS), the high valvulo-arterial impedance observed despite low valvular gradient suggests a high vascular load. Thoracic aortic calcifications (TACs) and valvular aortic calcifications (VACs) are, respectively, surrogates of aortic load and aortic valvular gradient. The aim of this study was to compare the respective contributions of TAC and VAC on 3-year cardiovascular (CV) mortality following TAVI in LGAS vs. highgradient aortic stenosis (HGAS) patients.

Methods

and results

A total of 1396 consecutive patients were included. TAC and VAC were measured on the pre-TAVI CT-scan. About 435 (31.2\%) patients had LGAS and 961 (68.8\%) HGAS. LGAS patients were more prone to have diabetes, coronary artery disease (CAD), atrial fibrillation (AF), and lower left ventricular ejection fraction (LVEF), $P<0.05$ for all. During the 3 years after TAVI, 245(17.8\%) patients experienced CV mortality, 92(21.6\%) in LGAS and $153(16.2 \%)$ in HGAS patients, $P=0.018$. Multivariate analysis adjusted for age, gender, diabetes, $A F, C A D, L V E F$, renal function, vascular access, and aortic regurgitation showed that TAC but not VAC was associated with CV mortality in LGAS, hazard ratio (HR) 1.085 confidence interval $(\mathrm{Cl})(1.019-1.156), P=0.011$, and $\mathrm{HR} 0.713 \mathrm{Cl}$ (0.439-1.8), $P=0.235$; the opposite was observed in HGAS patients with VAC but not TAC being associated with $\mathrm{CV}$ mortality, $\mathrm{HR} 1.342 \mathrm{Cl}(1.034-1.742), P=0.027$, and $\mathrm{HR} 1.015 \mathrm{Cl}(0.955-1.079), P=0.626$.

Conclusion TAC plays a major prognostic role in LGAS while VAC remains the key in HGAS patients. This confirms that LGAS is a complex vascular and valvular disease.

Keywords

TAVI • TAVR • aortic stiffness • vascular stiffness • outcome

\section{Introduction}

Low-gradient aortic stenosis (LGAS) is being more and more recognized as a peculiar form of aortic stenosis (AS); it currently represents $30-50 \%$ of patients with severe AS. ${ }^{1,2}$ The prognosis of LGAS is worse than that of high-gradient aortic stenosis (HGAS), the explanation being uncertain. ${ }^{2}$ In HGAS, the primary abnormality is valvular impediment represented by mean aortic gradient $\geq 40 \mathrm{mmHg}$, a surrogate of which may be valvular aortic calcifications (VACs). ${ }^{3,4}$ Despite a lower mean transaortic gradient, LGAS patients often exhibit a higher left ventricle afterload assessed by valvuloarterial impedance., We have shown that thoracic aortic

\footnotetext{
* Corresponding author. Tel: +33 (472) 071 668; Fax: +33 (472) 071 674. E-mail: brahim.harbaoui@chu-lyon.fr

t These authors take responsibility for all aspects of the reliability and freedom from bias of the data presented and their discussed interpretation.

Published on behalf of the European Society of Cardiology. All rights reserved. (c) The Author(s) 2021. For permissions, please email: journals.permissions@oup.com.
} 
calcifications (TACs), which reflects vascular impediment, are of prognosis significance after transcatheter aortic valve implantation $(\mathrm{TAVI})^{7,8}$; since our initial publications, this has been confirmed by other groups in other countries. ${ }^{9}$ We hypothesize that the contribution of VAC and TAC to the post-TAVI outcome may differ depending on aortic gradient: from a prevailing valvular disease in HGAS to a more complex valvular and vascular disease in LGAS.

Thus, the objective of this study was to compare the respective contribution of TAC and VAC on 3-year cardiovascular (CV) mortality following TAVI in LGAS as compared with HGAS patients.

\section{Methods}

\section{Patients}

Among the 1425 patients of the multicentric C4CAPRI study recruited between 2010 and 2014, 1396 patients with both an available preoperative CT-scan and a measurement of mean aortic gradient were included. The C4CAPRI cohort has been described elsewhere. ${ }^{10}$ Patients were indicated for TAVI in the presence of severe AS when surgical aortic valve replacement was either contraindicated or deemed at too high risk by the multidisciplinary Heart Team. Severe AS was defined by an aortic valve area $<1.0 \mathrm{~cm}^{2}$ (or $0.6 \mathrm{~cm}^{2} / \mathrm{m}^{2}$ ) and/or a mean transaortic pressure gradient $>40 \mathrm{mmHg}$ and/or a peak aortic jet velocity $(V \max )>4 \mathrm{~m} / \mathrm{s}$. Mean aortic gradient was obtained with transthoracic echography (TTE). TTE was performed by a senior cardiologist, using continuous Doppler in the most appropriate window among five apical chamber view, right parasternal, and suprasternal views. LGAS was defined as a mean aortic gradient $<40 \mathrm{mmHg}$ and HGAS as a mean aortic gradient $\geq 40 \mathrm{mmHg}$. The $\mathrm{C}_{4}$ CAPRI study was approved by the ethics committee (Comité de Protection des Personnes SUD-EST IV, L16-56) and by the Commission Informatique et Liberté (CNIL $N^{\circ}$ 16-065). All patients provided written informed consent to anonymous processing of their data.

\section{Patient and public involvement}

Before undergoing the TAVI procedures, patients were asked to give and sign an informed consent to participate to the registry.

Patients were not involved in the design of the study.

\section{Outcomes}

The primary outcome was CV mortality (according to the VARC-2 criteria $^{11}$ ) occurring within a 3-year follow-up period after TAVI. Vital status was obtained by telephone contact with patients, their relatives, carers or physicians, and by on-site planned visits. A follow-up was censored at 3year following TAVR. Two experienced cardiologists blinded to TAC, VAC, and to patient characteristics adjudicated CV mortality according to the VARC-2 criteria. ${ }^{11}$. Data collection was performed through dedicated web-based case report forms in each centre, which were merged for analysis. Range checks to identify extreme values and assessments of internal consistency were applied during upload.

\section{Measurement of TAC AND VAC}

The way measurements were performed has been described elsewhere. $^{10}$ Briefly, both TAC and VAC were extracted using semiautomated dedicated software from the CT scanner of the valve and the whole thoracic aorta with a very good reproducibility as previously described. ${ }^{10}$ For each patient, TAC was calculated from the aortic sinus to the aortic hiatus while excluding VAC, and VAC was measured
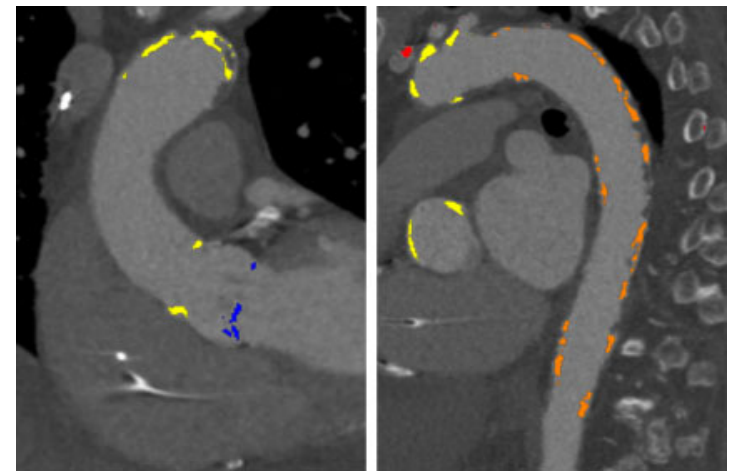

Figure I Example of calcifications measurement. Blue, yellow, and orange are for valvular, ascending, and descending thoracic aortic calcifications, respectively. Red calcifications do not belong to the aorta and have been excluded.

including valve leaflets and annular calcification while ignoring nonvalvular calcification in the left ventricular outflow tract, aortic sinus, coronary arteries, and mitral annulus. Figure 1 shows an example of VAC and TAC measurements.

\section{Statistical analysis}

Variables are summarized as means \pm standard deviations, or numbers and percentages, as appropriate. Comparisons between LGAS and HGAS patients were performed using $\chi^{2}$ test, unpaired $t$-test, or nonparametric test as appropriate. Both TAC and VAC were considered as categorical variables (three groups according to terciles of TAC and three groups according terciles of VAC) or continuous variables. Correlates of TAC and VAC were assessed using univariate and multivariate linear regression. Comparisons of distributions of TAC and VAC among LGAS and HGAS, patients were performed using a Mann-Whitney non-parametric test and illustrated using box plots.

The prognostic value of the TAC and VAC was first assessed by building Kaplan-Meier curves of cardiovascular mortality for the three groups defined according to the terciles of TAC and VAC. The three curves were compared using the log-rank test.

The prognostic value of both TAC and VAC considered as a continuous variable was further quantified and tested in univariate and multivariate Cox regression analysis.

Several models were built according to the existent literature.

- Model 1 adjusted for age and gender.

- Model 2 adjusted for Logistic EuroSCORE.

- Model 3 adjusted for age, gender, diabetes, atrial fibrillation, coronary artery disease, left ventricular ejection fraction (LVEF), and renal function.

- Model 4 adjusted for age, gender, diabetes, atrial fibrillation, coronary artery disease, LVEF, renal function, vascular access, and aortic regurgitation.

- Model 5 adjusted for age, gender, diabetes, atrial fibrillation, coronary artery disease, LVEF, renal function, vascular access, aortic regurgitation, and TAC or VAC as applicable. 
Table I Patient's baseline and procedural characteristics according to mean aortic gradient

\begin{tabular}{|c|c|c|c|}
\hline & LGAS Gradient $<40 \mathrm{mmHg}$ & HGAS Gradient $\geq 40 \mathrm{mmHg}$ & $P$ \\
\hline Number of patients & 435 & 961 & \\
\hline \multicolumn{4}{|l|}{ Demographic characteristics } \\
\hline Age (years) ${ }^{\mathrm{a}}$ & $82.7( \pm 7.5)$ & $83.7( \pm 6.7)$ & 0.01 \\
\hline Men, $n(\%)$ & $267(61.4 \%)$ & $448(46.6 \%)$ & $<0.001$ \\
\hline BMI $\left(\mathrm{kg} / \mathrm{m}^{2}\right)^{\mathrm{a}}$ & $26.1( \pm 4.7)$ & $26.4( \pm 5.3)$ & 0.3 \\
\hline \multicolumn{4}{|l|}{ Clinical history, $n$ (\%) } \\
\hline Diabetes & $129(29.7 \%)$ & $232(24.1 \%)$ & 0.035 \\
\hline Hypertension & 248 (71.7\%) & $589(71.5 \%)$ & 1 \\
\hline Smokers & $66(19 \%)$ & $126(15.2 \%)$ & 0.1 \\
\hline Dyslipidaemia & $180(52 \%)$ & $438(53.2 \%)$ & 0.7 \\
\hline Atrial fibrillation & $159(36.8 \%)$ & $287(30.2 \%)$ & 0.018 \\
\hline CAD & $222(51.3 \%)$ & $408(42.5 \%)$ & 0.002 \\
\hline PVD & $110(25.3 \%)$ & $200(20.8 \%)$ & 0.07 \\
\hline Previous stroke or TIA & 45 (10.4\%) & 79 (8.2\%) & 0.2 \\
\hline COPD & $90(20.7 \%)$ & $176(18.4 \%)$ & 0.3 \\
\hline NYHA 3/4 & $268(63.7 \%)$ & $582(61.8 \%)$ & 0.5 \\
\hline \multicolumn{4}{|l|}{ TTE parameters } \\
\hline $\operatorname{LVEF}(\%)^{\mathrm{a}}$ & $51.3( \pm 16.1)$ & $58.8( \pm 12.4)$ & $<0.001$ \\
\hline LVEF $<50 \%, n(\%)$ & $179(41.3 \%)$ & $196(20.5 \%)$ & $<0.001$ \\
\hline Mean aortic gradient $(\mathrm{mmHg})^{\mathrm{a}}$ & $30.3( \pm 6.6)$ & $54.7( \pm 13.4)$ & - \\
\hline Aortic valve area $\left(\mathrm{cm}^{2}\right)^{\mathrm{a}}$ & $0.74( \pm 0.21)$ & $0.63( \pm 0.17)$ & $<0.001$ \\
\hline Aortic valve area $\left(\mathrm{cm}^{2} / \mathrm{m}^{2}\right)^{\mathrm{a}}$ & $0.42( \pm 0.12)$ & $0.36( \pm 0.09)$ & $<0.001$ \\
\hline Moderate/severe MR, n (\%) & $7(1.6 \%)$ & $10(1.1 \%)$ & 0.4 \\
\hline $\operatorname{PASP}(\mathrm{mmHg})^{\mathrm{a}}$ & $43.7( \pm 15)$ & $43.8( \pm 14)$ & 0.8 \\
\hline \multicolumn{4}{|l|}{ Renal function } \\
\hline GFR $\left(\mathrm{mL} / \mathrm{min} / 1.73 \mathrm{~m}^{2}\right)^{\mathrm{a}}$ & $47.6( \pm 21.3)$ & $50.2( \pm 22.5)$ & 0.05 \\
\hline \multicolumn{4}{|l|}{ Euroscore $^{\mathrm{a}}$} \\
\hline & $19.18( \pm 11.6)$ & $16.77( \pm 9.79)$ & $<0.001$ \\
\hline \multicolumn{4}{|l|}{ Procedural and hospitalization } \\
\hline Femoral access $n(\%)$ & $306(70.5 \%)$ & $684(71.3 \%)$ & 0.7 \\
\hline Balloon expandable valve ( $n$ \%) & $278(64.1 \%)$ & $593(68.1 \%)$ & 0.2 \\
\hline Aortic Regurgitation >2 ( $n \%)$ & $3(0.7 \%)$ & $14(1.5 \%)$ & 0.3 \\
\hline Tamponade or annulus rupture ( $n \%)$ & $6(1.4 \%)$ & $33(3.5 \%)$ & 0.03 \\
\hline New pacemaker implantation & $66(18.6 \%)$ & $136(16.3 \%)$ & 0.3 \\
\hline
\end{tabular}

BMI, body mass index; CAD, coronary artery disease; COPD, chronic obstructive pulmonary disease; GFR, glomerular filtration rate; HGAS, high-gradient aortic stenosis; LGAS, low-gradient aortic stenosis; LVEF, left ventricular ejection fraction; MR, mitral regurgitation; NYHA, New York Heart Association; PASP, pulmonary artery systolic pressure; PVD, peripheral vascular disease; TIA, transient ischaemic attack; TTE, transthoracic echography.

${ }^{a} \mathrm{~V}$ alues are expressed as mean $\pm \mathrm{SD}$ or percentages.

Bold values $P<0.005$

All analyses were performed using SPSS software, release 20.0 .0 (SPSS, Chicago, IL, USA). A $P$-value $<0.05$ was considered statistically significant.

\section{Results}

\section{Baseline data}

Table 1 summarizes the characteristics of the cohort according to mean aortic gradient. Patients with LGAS were more prone to have diabetes, atrial fibrillation, renal dysfunction, coronary artery disease, lower LVEF, and higher logistic EuroSCORE $(P<0.05$ for all associations). HGAS patients experienced more procedural tamponade or annulus rupture than LGAS patients $(P=0.03)$.

Supplementary data online, Figure $S 1$ displays the distributions of TAC and VAC volumes among LGAS and HGAS patients. TAC was higher in LGAS patients $(P<0.001)$ compared with HGAS patients while VAC was higher in HGAS compared to LGAS patients $(P=0.027)$.

\section{Correlates of TAC AND VAC}

Table 2 summarizes the correlates of TAC and VAC in univariate and multivariate analyses. 
Table 2 Correlates of TAC and VAC: univariate and multivariate linear regression analysis

\begin{tabular}{|c|c|c|c|c|c|c|c|c|}
\hline \multirow[t]{3}{*}{ Variables } & \multicolumn{4}{|l|}{ TAC } & \multicolumn{4}{|l|}{ VAC } \\
\hline & \multicolumn{2}{|c|}{ Univariate } & \multicolumn{2}{|c|}{ Multivariate } & \multicolumn{2}{|c|}{ Univariate } & \multicolumn{2}{|c|}{ Multivariate } \\
\hline & $\boldsymbol{\beta}$ & $P$-value & $\beta$ & $P$-value & $\beta$ & $P$-value & $\beta$ & $P$-value \\
\hline Age (+1year) & 0.080 & 0.003 & 0.098 & 0.005 & 0.044 & 0.107 & & \\
\hline Gender (men) & 0.082 & 0.002 & 0.090 & 0.004 & 0.234 & $<0.001$ & 0.317 & $<0.001$ \\
\hline Hypertension (yes) & 0.174 & $<0.001$ & 0.149 & $<0.001$ & -0.053 & 0.081 & & \\
\hline Diabetes (yes) & 0.069 & 0.01 & 0.028 & 0.353 & -0.071 & 0.011 & -0.05 & 0.08 \\
\hline Dyslipidemia (yes) & 0.085 & 0.004 & 0.051 & 0.102 & 0.025 & 0.417 & & \\
\hline Smoking (yes) & 0.05 & 0.086 & & & 0.068 & 0.023 & 0.001 & 0.980 \\
\hline Coronary artery disease (yes) & 0.150 & $<0.001$ & 0.089 & 0.004 & -0.043 & 0.118 & & \\
\hline Peripheral vascular disease (yes) & 0.170 & $<0.001$ & 0.123 & $<0.001$ & -0.061 & 0.028 & -0.068 & 0.019 \\
\hline Atrial fibrillation (yes) & 0.033 & 0.217 & & & 0.005 & 0.844 & & \\
\hline Estimated glomerular filtration rate $(+1 \mathrm{~mL} / \mathrm{min})$ & -0.111 & $<0.001$ & -0.086 & 0.013 & 0.022 & 0.441 & & \\
\hline Left ventricular ejection fraction $(+1 \%)$ & -0.028 & 0.290 & & & -0.005 & 0.868 & & \\
\hline Aortic-valve area $\left(+1 \mathrm{~cm}^{2}\right)$ & 0.040 & 0.139 & & & -0.096 & 0.001 & -0.055 & 0.075 \\
\hline Mean aortic valve gradient $(+1 \mathrm{mmHg})$ & -0.056 & 0.037 & 0.017 & 0.590 & 0.217 & $<0.001$ & & $<0.001$ \\
\hline $\operatorname{LogTAC}\left(+1 \mathrm{~cm}^{3}\right)$ & & & & & 0.022 & 0.435 & & \\
\hline $\operatorname{LogVAC}\left(+1 \mathrm{~mm}^{3}\right)$ & 0.022 & 0.435 & & & & & & \\
\hline
\end{tabular}

TAC, thoracic aorta calcifications; VAC, valvular aortic calcifications. Bold values $P<0.005$

In multivariate analyses, the correlates of TAC were age, gender, hypertension, coronary artery disease, peripheral vascular disease, and renal function, $P<0.05$ for all. In multivariate analyses, the correlates of VAC were gender, mean aortic gradient, and to a lesser extent peripheral vascular disease, $P<0.05$ for all.

\section{Prognostic value of TAC AND VAC}

After 3 years of follow-up, 23 (1.6\%) patients were lost to follow-up and 245 (17.8\%) patients experienced CV mortality, 92 (21.6\%) in LGAS patients, and 153 (16.2\%) in HGAS patients, respectively, pLogRank=0.018 (Figure 2).

Figure 3 displays the Kaplan-Meier curves according to the three groups defined according to the terciles of TAC and VAC according to mean aortic gradient. TAC ranges were $0-1.359 \mathrm{~cm}^{3}$ for Group 1, $1.36-3.616 \mathrm{~cm}^{3}$ for Group 2, and 3.619-22.33 $\mathrm{cm}^{3}$ for Group 3. VAC ranges were 0-573.6 $\mathrm{mm}^{3}$ for Group 1, 573.7-1077 $\mathrm{mm}^{3}$ for Group 2, and 1078-4517.28 $\mathrm{mm}^{3}$ for Group 3. In LGAS patients, the survival curves of the three groups defined according to terciles of TAC were significantly different for CV mortality ( $p$ LogRank=0.007) (Figure $3 \mathrm{~A}$ ) with a survival much lower for patients belonging to the third group; conversely, no difference of the survival curves of the three groups defined according to terciles of VAC (Figure 3B) was observed. In HGAS, the survival curves of the three groups defined according to terciles of both TAC and VAC were significantly different for CV mortality (pLogRank=0.04 and 0.005, respectively) (Figure 3C and D). Again the survival was lower for patients belonging to the third groups defined according to terciles of TAC or VAC.

Table 3 summarizes univariate and multivariate Cox analyses in LGAS and HGAS patients.

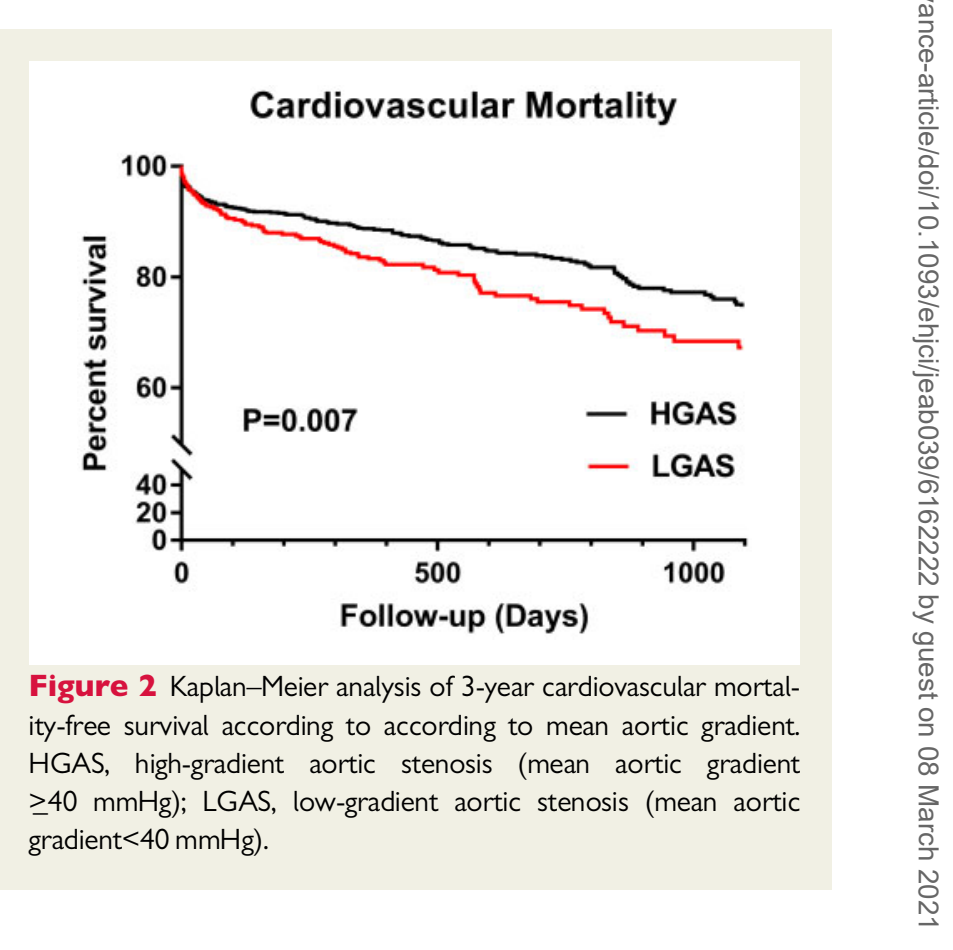

In LGAS patients, TAC was associated with CV mortality in univariate analysis and in all multivariate models while VAC was not. In HGAS patients, TAC remained only associated with CV mortality in univariate analysis and after adjustment for age and gender but not in other multivariate models, while VAC was associated with CV mortality in univariate and in all multivariate models. 

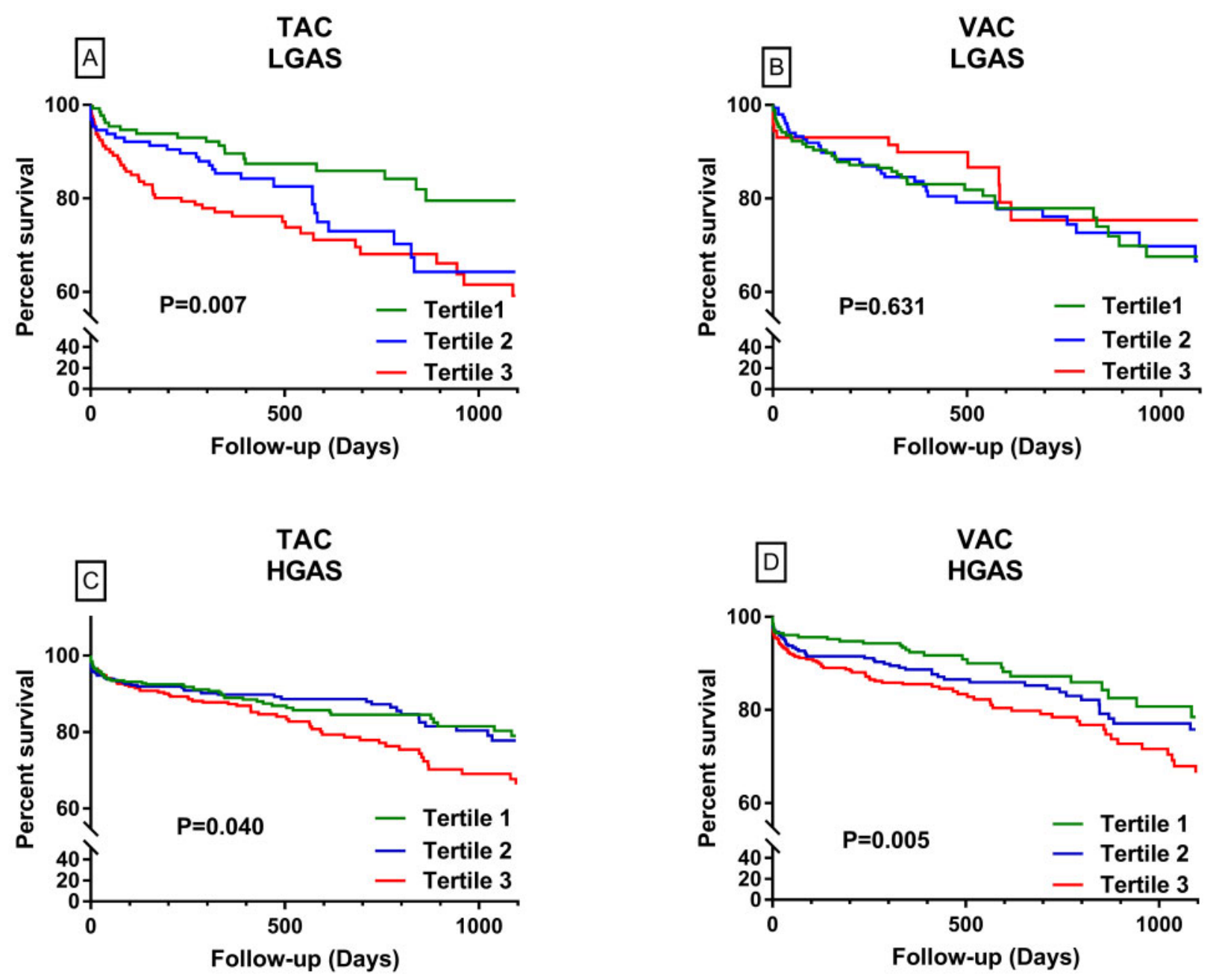

Figure 3 Kaplan-Meier analysis of 3-year cardiovascular mortality-free survival according to groups defined on terciles of TAC and VAC in LGAS and HGAS patients. HGAS, high-gradient aortic stenosis (mean aortic gradient $\geq 40 \mathrm{mmHg}$ ); LGAS, low-gradient aortic stenosis (mean aortic gradient $<40 \mathrm{mmHg}$ ); TAC, thoracic aortic calcifications; $V A C$, valvular aortic calcifications.

\section{Discussion}

By considering the prognostic influence of VAC and TAC on postTAVI outcomes, we highlighted some pathophysiological differences between LGAS and HGAS patients; while the prognosis of the classical HGAS form is mainly driven by valvular aspects, which of LGAS is more complex, being related to vascular features. This explains, at least in part, why both AS forms do not benefit to the same extent from a TAVI procedure and suggest that TAC and VAC should be considered for risk stratification.

This study confirms that LGAS and HGAS patients are different with respect to some important clinical characteristics and comorbidities. LGAS also have a worse prognosis than HGAS after valve replacement in line with previous reports. ${ }^{12}$ The course of the disease could be influenced by factors that may differ between LGAS and HGAS patients. Traditional AS is characterized by a severe reduction of aortic valve area, correlated to both a high VAC and a high gradient, ${ }^{3,4}$ as confirmed in this study. HGAS is an unequivocal valvular disease as emphasized by the major prognostic significance of VAC in this this situation. This is consistent with previously published studies in patients with AS, treated by TAVI or surgery, demonstrating that VAC was associated with mortality. ${ }^{13}$ The mechanisms underlying the association of VAC with post-TAVI outcomes may be linked to an increased rate of periprocedural complications, device failure, pacemaker implantation, or paravalvular leak. ${ }^{14,15}$ AS severity, and thus VAC, is also correlated with myocardial fibrosis, ${ }^{16}$ which is mostly irreversible and influences post-operative mortality ${ }^{17}$ and heart failure. ${ }^{18}$ This may explain that VAC continued to have a prognostic significance over the long run, i.e. after AS relief.

In LGAS patients, TAC but not VAC represents a major risk factor for CV mortality. Albeit TAC and VAC share some common pathways, ${ }^{19}$ both being linked with atherosclerotic risk factors, they are not tightly related suggesting different significance. TAC seems more integrative, being related to CV factors, CV disease, and renal function. In this respect, the prognostic impact of TAC in LGAS is in line with a more diffuse disease encompassing cardiac, vascular, and 
Table 3 Risk of 3-year cardiovascular mortality in unadjusted and adjusted Cox regression models

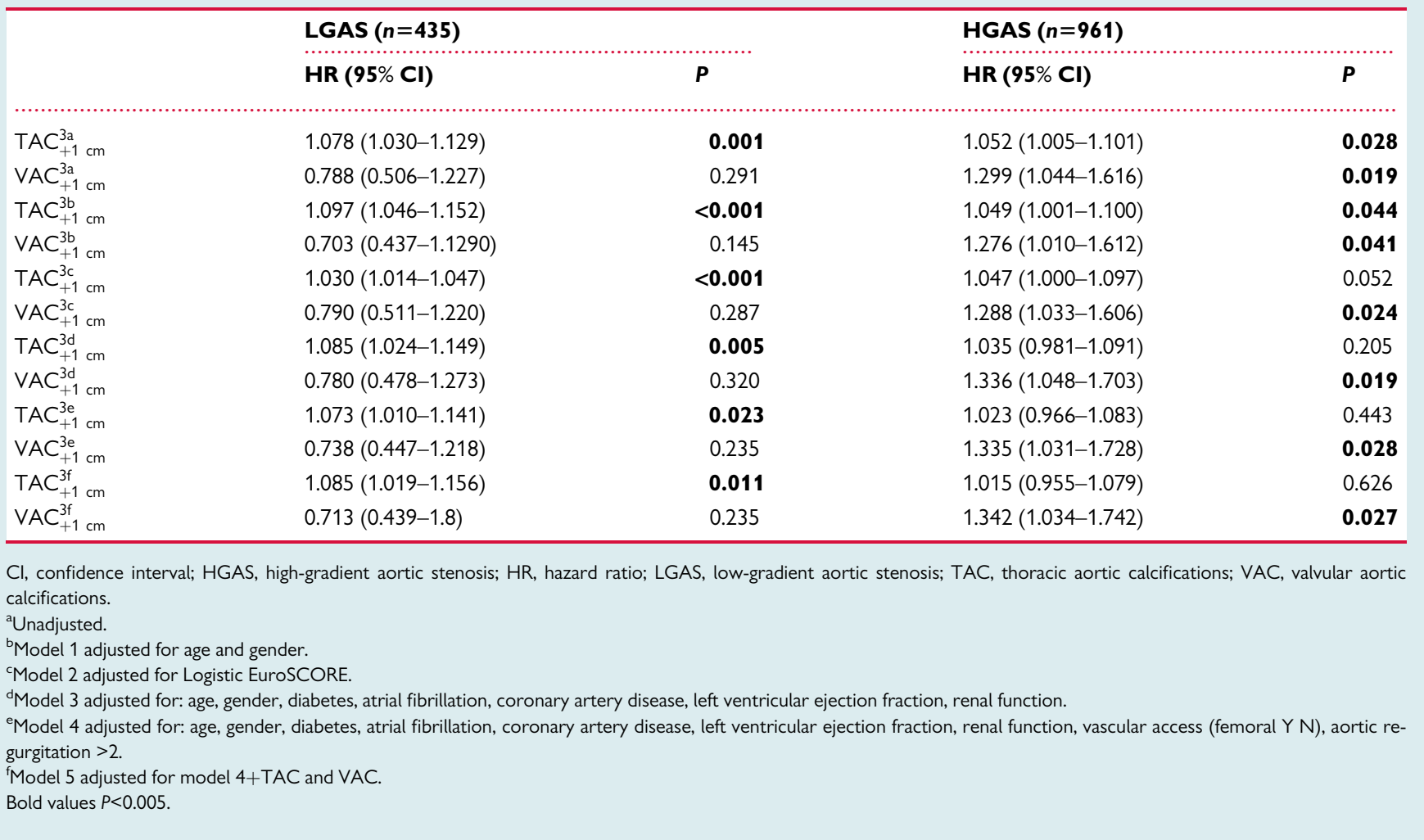

kidney damages. Moreover, TAC represents a surrogate of vascular afterload ${ }^{20}$; it has been shown that the negative prognosis impact of an increased vascular load was even worse in the presence of low LVEF or low stroke volume. ${ }^{5,6}$ In the setting of TAVI, the high residual vascular load will continue to exert its detrimental influence on left ventricular function over the long time. ${ }^{7,8,21}$ This is in line with a lower left ventricle mass regression and a lower LVEF improvement at 1-year follow-up in AS patients with a high calcification burden of the thoracic aorta. ${ }^{22}$ TAC has recently been incorporated in a dedicated TAVI risk score, the CAPRI score, that predicts 1-year mortality $^{10}$ and hospitalization for heart failure. ${ }^{23}$. The fact that VAC was not of prognostic importance in this setting, reinforces the view that, valvular disorder is not the sole component of LGAS.

\section{Clinical implications}

The present multicentric study highlights the pathophysiological differences existing between LGAS and HGAS patients. The preTAVI CT-scan provides some crucial prognostic information by quantifying the calcification load of both the thoracic aorta and the valve. On top of these pathophysiological insights, this study has important prognostic consequences. TAC may be crucial to consider for risk stratification, particularly in the setting of LGAS to foresee the post-TAVI outcome. On top of being associated with peri-procedural complications, VAC will still influence outcome after restoration of transvalvular flow in HGAS patients. Nowadays, VAC and TAC are easily measurable with CT-scan, and artificial intelligence will probably allow to fully automatize their measurements as it is already the case for coronary calcifications. Therefore, together with other predictors of outcomes,
VAC and TAC may help in the decision-making process during the TAVI work-up with the aim of selecting patients with a high probability of poor outcome after the procedure.

\section{Limits}

The present ancillary study of C4CAPRI study is a registry, even though the completeness of data entered is high, ${ }^{10}$ we may not exclude that some information may not have been available in the database. For example, the repeatability of Doppler data was not assessed.

With the improvement of aortic valve bioprosthesis, and the evolution of TAVI indications, the patients treated nowadays by TAVI may not fully match in terms of risk with those included in the present study, i.e. between 2010 and 2014, questioning the generalizability of the study results. Still, valvular and aortic calcifications are common findings in the AS setting and will still be encountered in lower risk patients. VAC and left ventricle outflow tract calcifications have been associated with more aortic regurgitation and more pacemaker implantation after TAVI. While new generation prosthesis has partly addressed these drawbacks, it is unlikely that their use would lessen the prognostic significance of VAC; indeed the rate of aortic regurgitation is already low in the study population, and, in any case, not different between the more calcified HGAS patients than in the LGAS ones. Thresholds of VAC volume have been proposed in order to stratify the severity of AS with a special attention to gender. However, these thresholds have been determined on a different CTScan protocol. In our work, the aim was not to define a threshold as all patients were considered to have a severe AS. The aim was to highlight the differential prognostic value of TAC and VAC in HGAS and LGAS patients. It would have been interesting to assess the 
stroke volume index in order to better categorize LGAS patients; however, in this study, we only have the LVEF which is to some extent a surrogate. Finally, a lack of statistical power in HGAS patients (less events) may explain the absence of prognostic value of TAC in the full adjusted model.

\section{Conclusions}

TAC and VAC should be carefully analysed and quantified during the pre-TAVI CT-scan since they carry specific prognostic information in LGAS and HGAS patients, corresponding to partly different underlying pathological mechanisms.

\section{Supplementary data}

Supplementary data are available at European Heart Journal - Cardiovascular Imaging online.

\section{Data availability}

Possible, please send an email to the corresponding author with the purpose of your demand.

Conflict of interest: No conflict of interest regarding the present work.

\section{References}

1. Minners J, Allgeier M, Gohlke-Baerwolf C, Kienzle RP, Neumann FJ, Jander N. Inconsistencies of echocardiographic criteria for the grading of aortic valve stenosis. Eur Heart J 2008;29:1043-8.

2. Clavel MA, Magne J, Pibarot P. Low-gradient aortic stenosis. Eur Heart J 2016;37: 2645-57.

3. Clavel MA, Messika-Zeitoun D, Pibarot P, Aggarwal SR, Malouf J, Araoz PA et al. The complex nature of discordant severe calcified aortic valve disease grading: new insights from combined Doppler echocardiographic and computed tomographic study. J Am Coll Cardiol 2013;62:2329-38.

4. Pawade T, Sheth T, Guzzetti E, Dweck MR, Clavel MA. Why and how to measure aortic valve calcification in patients with aortic stensis. JACC Cardiovasc Imaging 2019;12:1835-48.

5. Hachicha Z, Dumesnil JG, Bogaty P, Pibarot P. Paradoxical low-flow, low-gradient severe aortic stenosis despite preserved ejection fraction is associated with higher afterload and reduced survival. Circulation 2007;115:2856-64.

6. Cramariuc D, Cioffi G, Rieck AE, Devereux RB, Staal EM, Ray S et al. Low-flow aortic stenosis in asymptomatic patients: valvular-arterial impedance and systolic function from the SEAS Substudy. JACC Cardiovasc Imaging 2009;2:390-9.
7. Harbaoui B, Courand PY, Charles P, Dauphin R, Boussel L, Jegaden O et al. Aortic calcifications present the next challenge after TAVR. J Am Coll Cardiol 2015;65:1058-60

8. Harbaoui B, Montoy M, Charles P, Boussel L, Liebgott H, Girerd N et al. Aorta calcification burden: towards an integrative predictor of cardiac outcome after transcatheter aortic valve implantation. Atherosclerosis 2016;246:161-8.

9. Gegenava T, Vollema EM, Abou R, Goedemans L, van Rosendael A, van der Kley $F$ et al. Prognostic value of thoracic aorta calcification burden in patients treated with TAVR. JACC Cardiovasc Imag 2019;12:216-7.

10. Lantelme P, Eltchaninoff H, Rabilloud M, Souteyrand G, Dupre M, Spaziano M et al Development of a risk score based on aortic calcification to predict 1-year mortality after transcatheter aortic valve replacement. JACC Cardiovasc Imaging 2019;12:123-32.

11. Kappetein AP, Head SJ, Genereux P, Piazza N, van Mieghem NM, Blackstone EH et al. Updated standardized endpoint definitions for transcatheter aortic valve implantation: the Valve Academic Research Consortium-2 consensus document. J Am Coll Cardiol 2012;60:1438-54.

12. Clavel MA, Dumesnil JG, Capoulade R, Mathieu P, Senechal M, Pibarot P. Outcome of patients with aortic stenosis, small valve area, and low-flow, lowgradient despite preserved left ventricular ejection fraction. J Am Coll Cardiol 2012;60:1259-67.

13. Clavel MA, Pibarot P, Messika-Zeitoun D, Capoulade R, Malouf J, Aggarval S et al. Impact of aortic valve calcification, as measured by MDCT, on survival in patients with aortic stenosis: results of an international registry study. J Am Coll Cardiol 2014;64:1202-13.

14. John D, Buellesfeld L, Yuecel S, Mueller R, Latsios G, Beucher $H$ et al. Correlation of device landing zone calcification and acute procedural success in patients undergoing transcatheter aortic valve implantations with the selfexpanding CoreValve prosthesis. JACC Cardiovasc Interven 2010;3:233-43.

15. Akodad M, Lattuca B, Agullo A, Macia JC, Gandet T, Marin G et al. Prognostic impact of calcium score after transcatheter aortic valve implantation performed with new generation prosthesis. Am J Cardiol 2018;121:1225-30.

16. Song I, Ko SM, Yi JG, Chee HK, Kim JS. Differences in aortic valve and left ventricular parameters related to the severity of myocardial fibrosis in patients with severe aortic valve stenosis. PloS One 2017;12:e0170939.

17. Milano AD, Faggian G, Dodonov M, Golia G, Tomezzoli A, Bortolotti U et al. Prognostic value of myocardial fibrosis in patients with severe aortic valve stenosis. J Thorac Cardiovasc Surg 2012;144:830-7.

18. Weidemann F, Herrmann S, StöRk S, Niemann M, Frantz S, Lange $V$ et al. Impact of myocardial fibrosis in patients with symptomatic severe aortic stenosis. Circulation 2009:120:577-84.

19. Pawade TA, Newby DE, Dweck MR. Calcification in aortic stenosis: the skeleton key. I Am Coll Cardiol 2015;66:561-77.

20. McEniery CM, McDonnell BJ, So A, Aitken S, Bolton CE, Munnery M et al. Aortic calcification is associated with aortic stiffness and isolated systolic hypertension in healthy individuals. Hypertension 2009;53:524-31.

21. Yotti R, Bermejo J, Gutierrez-lbanes E, Perez del Villar C, Mombiela T, Elizaga et al. Systemic vascular load in calcific degenerative aortic valve stenosis: insight from percutaneous valve replacement. J Am Coll Cardiol 2015;65:423-33.

22. Gegenava T, Vollema EM, van Rosendael A, Abou R, Goedemans L, van der Kley $\mathrm{F}$ et al. Changes in left ventricular global longitudinal strain after transcatheter aortic valve implantation according to calcification burden of the thoracic aorta. J Am Soc Echocardiogr 2019;32:1058-66 e2.

23. Harbaoui B, Durand E, Dupre M, Rabilloud M, Souteyrand G, Courand PY et al. Significance of the CAPRI risk score to predict heart failure hospitalization postTAVI: the CAPRI-HF study. Int J Cardiol 2019;296:98-102. 